\title{
Las mujeres y los regímenes de bienestar. Una mirada feminista para el debate de la organización social del cuidado en Argentina
}

Women and welfare regimes. A feminist perspective to assess the social organization of care in Argentina

Gisela Cánovas Herrera

Socióloga (Universidad de Buenos Aires). Docente de la Universidad Nacional de Hurlingham; Maestranda en Políticas Públicas y Gerenciamiento del Desarrollo (Universidad Nacional de San Martin/Georgetown University). Campo de trabajo: políticas educativas; políticas de cuidado.

giselapcanovas@gmail.com

\section{Resumen}

El objetivo de este trabajo se enmarca en los análisis de la organización social del cuidado en Argentina. En primer lugar, se recorren los debates en torno a los estudios de bienestar y el aporte del feminismo. En segundo lugar, se abordan las discusiones actuales sobre el cuidado, para luego utilizarlo como una herramienta analítica de las políticas sociales. Específicamente, se abordará el caso de la Asignación Universal por Hijo (AUH), para indagar los supuestos relacionados con el cuidado y el rol de las mujeres que subyacen a esta política pública. Finalmente, se exploran los posibles efectos que tienen estos supuestos en la participación laboral de las mujeres en Argentina.

Palabras clave: bienestar - distribución del cuidado - economía feminista - trabajo femenino Asignación Universal por Hijo.

Fecha de recepción:

15.2.18

\section{Abstract}

The aim of this work is to analyze the social organization of care in Argentina. In the first place, we study the debates about welfare studies and how feminism

29.6.18 
contributes on this issue. Then, we address current discussions on care, and how it can be used as an analytical tool for social policies. Specifically, we analyze the case of the Universal Assignment by Child (AUH), to explore assumptions about care and role of women that underlie this public policy. Finally, we explore the possible effects of these assumptions on women's labor participation.

Key-words: wellbeing - care regimes - feminist economy - women's work - Universal allowance per child.

\title{
Las mujeres y los regímenes de bienestar. Una mirada feminista para el debate de la organización social del cuidado en Argentina
}

\author{
"Nos ha nacido un niño"
}

\begin{abstract}
A principios de año, Laura fue mamá. ${ }^{1}$ Luego de visitarla en el sanatorio, dejé pasar un par de meses para volver a verla, para no invadir ese primer momento tan íntimo y agotador. Una de las cosas que me contó, resignada, fue que al principio llegó a no bañarse por cuatro días seguidos porque el padre de la criatura no quería quedarse a solas con la recién nacida ni por 15 minutos.
\end{abstract}

Hace un tiempo se reincorporó al trabajo. Con su pareja, decidieron que no iban a dejar a la niña en una guardería. Por un lado, porque no estaban seguros de la calidad del cuidado que le iban a brindar. No la iban a cuidar como ellos lo hacen. Por otro lado, porque las guarderías son muy costosas y su situación económica se los dificulta. Finalmente, se acordó una estrategia familiar de cuidado: las respectivas madres de la pareja se turnan para cuidar a la pequeña mientras ellos trabajan.

\section{Lo personal es político}

En los años sesenta, el feminismo de la segunda ola sacó los trapitos al sol. Prendió los reflectores e iluminó aquellas áreas de la vida que hasta ese momento se consideraban “privadas” y las identificó como epicentros de la dominación. Revolucionó la teoría política al analizar las relaciones de poder que estructuran la familia y la sexualidad. Visibilizó las conexiones que existen entre las experiencias personales de las mujeres y las grandes estructuras sociales y políticas. Nos permitió entender que aquello que cada una de nosotras percibe como algo aislado e individual, en realidad también forma parte de las trayectorias vitales de muchas otras mujeres. 
El objetivo de este trabajo se enmarca en los análisis de la organización social del cuidado en Argentina. Nuestra "puerta de entrada” para el análisis será la organización del cuidado infantil en nuestro país. Esto nos permitirá, siguiendo a Esquivel (2012: 157), una mirada transversal, que nos develará "un caleidoscopio de políticas, programas y regulaciones (...), no necesariamente coherentes entre sí (...) que se descubren como 'capas geológicas' de la política social".

Para esto, realizaremos un recorrido que pasará primero por los debates en torno a los estudios de bienestar y el aporte del feminismo al estudio de los regímenes. Luego nos adentraremos en las discusiones actuales sobre el cuidado y la conformación de un campo de estudio alrededor de este concepto, para luego utilizarlo como una herramienta analítica de las políticas sociales. Más específicamente, nos interesa iluminar el "detrás de escena” de la Asignación Universal por Hijo (AUH), ${ }^{2}$ preguntándonos por los supuestos relacionados con el cuidado y el rol de las mujeres que subyacen a esta política pública. Finalmente, exploraremos los posibles efectos que tienen estos supuestos en la participación laboral de las mujeres.

\section{Los regímenes de bienestar y la mirada feminista}

"Uno de los instrumentos más eficaces del gobierno patriarcal es el dominio económico que ejerce sobre las mujeres. (...) Ya que en las sociedades patriarcales la mujer siempre ha trabajado, realizando con frecuencia las tareas más rutinarias o pesadas, el problema central no gira en torno al trabajo femenino, sino a su retribución económica"

Kate Millet, Política Sexual

Hasta la década de 1990, el bienestar fue abordado principalmente en su dimensión económica. Esto implicó considerar a la generación de ingresos y a la disponibilidad de servicios sociales para la población como las fuentes principales del bienestar. Al considerar al trabajador asalariado como el sujeto central de la política pública, el bienestar se vinculó a la inserción ocupacional. De esta manera, los Estados de Bienestar se configuraron sobre la base de un modelo familiar de varón proveedor-mujer ama de casa. La esposa y los/as niños/as gozaban de derechos sociales en tanto y en cuanto eran “dependientes" del jefe de hogar (Faur 2014).

Esta mirada no permitía visibilizar de qué manera se lograba la sostenibilidad de la vida, es decir tanto la gestión de la vida y de los cuerpos, aquello que permite sostener la fuerza de trabajo, como la gestión de los aspectos ligados a la salud emocional, fisica y psicológica de los sujetos que la integran e integrarán esa fuerza. El trabajo doméstico y el cuidado de niños/as, adultos mayores y enfermos eran funcionales tanto a los mercados de trabajo como a la provisión de servicios sociales públicos. 
En la década de 1990 se da un punto de inflexión cuando Gosta Esping-Andersen postula la noción de "régimen de bienestar", planteando que la producción de bienestar no atañe de forma exclusiva a las políticas estatales, sino que es necesario analizar de qué manera se distribuyen las responsabilidades sociales entre el Estado, el mercado y la familia (la "tríada del bienestar") (Sunkel 2007).

La manera en la que se combinan estos tres elementos da como resultado dos procesos de independencia o autonomía del bienestar de las familias y las personas: la desmercantilización, que se refiere al grado en el que el acceso al bienestar se distancia de la participación de las personas en el mercado; y la desfamiliarización, que se refiere al grado en el que el bienestar deja de ser exclusiva responsabilidad de la familia (y por lo tanto de las mujeres).

Esping-Andersen identifica tres tipos ideales de regímenes de bienestar europeos (Draibe y Riesco 2006):

1) Régimen socialdemócrata (países escandinavos), el cual se funda en la solidaridad de base universal y el Estado desempeña un papel central en la previsión social, frente a las posiciones marginales de la familia y del mercado.

2) Régimen liberal (países anglosajones), el cual se funda en la base individual de la solidaridad y el mercado tiene un rol central en la previsión social, frente a los roles marginales de la familia y del Estado.

3) Régimen conservador-corporativo (países de Europa continental), el cual se funda en la solidaridad familiar y la familia desempeña un papel central en la previsión social, frente al carácter marginal del mercado y el Estado.

Si se analiza la combinación de los tres elementos de la tríada de bienestar, puede decirse que el régimen de bienestar socialdemócrata presenta el grado máximo de desmercantilización e incentiva la desfamiliarización, mientras que el régimen de bienestar liberal presenta grados mínimos de desmercantilización y un familismo implícito. A media distancia queda el régimen conservador, el cual presenta un alto grado de desmercantilización para el hombre proveedor y es explícitamente familista, ya que considera que, asegurado el ingreso, la familia podrá hacerse cargo de la mayoría de las funciones relacionadas con el bienestar (Sunkel 2007).

Las críticas feministas contribuyen a reconocer y visibilizar el papel constitutivo de las relaciones de género en las estructuras y efectos del estado de bienestar. Al configurarse sobre la base de un tipo de familia patriarcal, la posición social de la mujer en los regímenes de bienestar tiene dos caras: por un lado depende del hombre para su manutención, pero por otro lado tiene a su cargo el cuidado de todos los miembros de su familia. 
De esta manera, en las sociedades capitalistas la producción de bienestar no se hace sólo a través del intercambio mercantil y de la presencia del Estado, sino que también a través del trabajo no remunerado, principalmente realizado por las mujeres, en el marco de las familias (Draibe y Riesco 2006).

Los regímenes de bienestar re-estratifican (reubican a las personas en términos socio-económicos y genéricos), pero también, dependiendo de cómo se combinen sus elementos, pueden promover condiciones distributivas más o menos favorables que las que permiten el intercambio mercantil y la división sexual del trabajo (Martínez Franzoni 2005).

\section{Los regímenes de bienestar en América Latina}

\section{a) Movimientos en las estructuras de bienestar latinoamericanas}

Durante la década de 1980, y posterior a la crisis de la deuda de 1982, la gran mayoría de los países de América Latina atravesaron un proceso de transición a gobiernos electos de manera democrática. Cavarozzi (2017) denomina a este momento como panacea democrática, que se basó en el supuesto de que la vigencia de la soberanía popular resolvería los problemas políticos y económicos que enfrentaban las sociedades en la región.

Años después, los proyectos reformistas y estatistas asociados a los primeros gobiernos democráticos fracasaron estrepitosamente. Argentina, Perú, Bolivia y Brasil ingresaron en la hiperinflación y los partidos que estaban en el gobierno perdieron las elecciones.

En los años noventa predominó el enfoque de mercado y reformas económicas inspiradas por el Consenso de Washington y los organismos multilaterales. Esta fase ascendente de "magia del mercado" se extendió hasta 1998/ 1999, momento en el cual América Latina fue duramente golpeada por la crisis mundial que se había iniciado en el sudeste asiático y en Rusia (Cavarozzi 2017). La consecuencia fue el mantenimiento o incremento de la pobreza y la desigualdad previa.

Estas reformas estructurales implicaron un cambio en el paradigma organizador de las políticas públicas de la seguridad social. El Estado pierde protagonismo y el mercado se constituye en un actor central de la tríada. En palabras de Sunkel (2007), "el nuevo sistema se basa en una concepción individual del sujeto que aporta a su bienestar y en el que, en base a sus ingresos, podrá extender la red de beneficios a su familia”.

Estos procesos erosionaron un sistema de bienestar constituido durante el siglo XX y que tenía sus pilares fundamentales en la protección y otorgamiento de derechos de ciudadanía a través de la participación en el mercado laboral. Se alteró el equilibrio de la tríada de bienestar y el rol cada vez más protagónico del mercado frente al Estado 
mercantilizó los servicios públicos de salud, educación, vivienda, jubilaciones y pensiones. Ante un Estado que intervenía sólo con programas focalizados y financiados por organismos internacionales, las familias y las redes comunitarias tuvieron que absorber este impacto regresivo (Faur 2014).

En este contexto de empobrecimiento y fracturas de los lazos sociales, se observa un llamativo aumento de la participación femenina en el mercado laboral al mismo tiempo que aumentaba la desocupación masculina. En los años en los que la crisis llegó a su punto máximo, la ocupación femenina fue más alta que la masculina en los sectores de más bajos ingresos. Así, los ingresos de las mujeres muchas veces se configuraron como el sostén principal de sus hogares. ${ }^{4}$

Al mismo tiempo que ingresaban a un mercado laboral sumamente debilitado y precarizado, las mujeres más pobres se enfrentaron con el aumento de la importancia de la actividad doméstica no remunerada (por ejemplo, en vez de comprar productos en el mercado se los producía en el hogar, se pasaba mucho tiempo buscando mejores precios de los productos básicos) para llegar a fin de mes.

Entonces las mujeres, al mismo tiempo que comenzaron a participar de manera masiva en la esfera laboral, convirtiéndose muchas de ellas en jefas de hogar por ser el sostén principal frente a la creciente desocupación masculina, se volvieron actoras claves y fundamentales en la supervivencia de los miembros de sus hogares y comunidades al aumentar y multiplicar su intervención en los ámbitos productivo, reproductivo y comunitario.

Pero estos procesos ¿suponían la transformación de la situación subordinada de las mujeres? Faur plantea que, en lugar de imaginar e implementar estrategias que permitan superar las desigualdades existentes, las políticas y los programas sociales focalizados de aquellos años afianzaron la mirada maternalista en las tareas domésticas y de cuidado social al valerse de las capacidades y solidaridades femeninas para cubrir las necesidades materiales más inmediatas de los hogares pobres (por ejemplo, el sostenimiento de comedores populares).

\section{b) Pensar el bienestar desde el Sur}

En estos años se produjo una serie de quiebres políticos que fueron más allá de una alternancia de partidos en el gobierno. Se agotaron sistemas de partidos establecidos y emergieron liderazgos asociados a clases más postergadas, como Lula da Silva en Brasil y Evo Morales en Bolivia. Durante los primeros años del siglo XXI, se revitalizó una versión ampliada de Estado de Bienestar, que impulsó políticas de transformación social y un recambio en el área de los programas sociales. El Estado volvió a tener un rol protagónico, aunque esto no implicó un debilitamiento de la mercantilización de los servicios públicos. 
En este marco, América Latina atraviesa un proceso de transición demográfica que moldea las prácticas de asignación de recursos y producción del bienestar. Por un lado, la proporción de personas dependientes de un único ingreso ha disminuido. Esto quiere decir que con el ingreso masivo de mujeres al mercado laboral entra en crisis la familia nuclear de hombre único proveedor, sobre la cual se erigió el edificio de la previsión social.Al mismo tiempo, las jefaturas femeninas han aumentado. Esto se combina con un aumento de la soltería, las separaciones y los divorcios, tanto como producto del aumento de la esperanza de vida como de las migraciones. Respecto de esto, la región se caracteriza por altas corrientes migratorias que impactan en la fragmentación de las familias, acentuando la división del trabajo entre quienes generan ingresos y quienes cuidan a niños/as y adultos/as mayores. Finalmente, las encuestas de uso del tiempo disponible (Marco Navarro 2012) muestran que el trabajo no remunerado continúa estando casi únicamente en manos de mujeres. ${ }^{5}$

Las transformaciones en los programas sociales que ocurrieron en Argentina durante los años de kirchnerismo (2003-2015) complejizaron el sistema de protección, superponiendo viejos y nuevos modelos de política social. Así, la lógica de bienestar de nuestro país es diversificada y plural, y dificil de analizar utilizando los "tipos ideales" de los regímenes de bienestar europeos.

Por eso se volvió necesario “pensar desde el Sur”. Martínez Franzoni (2005) se hace la pregunta de cómo y por qué pensar el bienestar en América Latina. Las sociedades de estos países identifican a la política y a las políticas públicas como las responsables de la pobreza, lo que vulnera la consolidación de la democracia. Por eso, dice la autora, urge la necesidad de construir una "nueva arquitectura" del bienestar que logre dar cuenta de la reconfiguraciones sufridas por América Latina en las últimas décadas. Esto conlleva "profundas implicaciones éticas, valorativas y económicas con respecto a la sociedad deseable y a la sociedad posible”.

En este escenario poco alentador, Martínez Franzoni identifica tres principales regímenes de bienestar en América Latina, que no logran reflejar el momento transicional en el que se encuentra la región:

1) Estatal de proveedor único. El Estado continúa asignando la mayor parte de sus recursos a servicios universales, y prevalece la división sexual del trabajo entre hombres proveedores y mujeres cuidadoras. Este régimen tiene la menor desigualdad socioeconómica de la región (coeficientes de Gini menores a .50).

2) Liberal de proveedor único. Este régimen es similar al anterior en cuanto a la división sexual tradicional del trabajo pero se verifica un desplazamiento desde el Estado hacia la prestación privada de servicios, en particular de salud, educación y pensiones. Presenta una desigualdad socioeconómica alta (coeficientes de Gini de .55 o más). 
3) Informal de doble proveedor. Las mujeres deben participar del mercado laboral para alcanzar niveles mínimos de ingresos porque las familias son de doble proveedor. Al mismo tiempo, las familias absorben el grueso de la producción de bienestar. Presenta niveles de desigualdad socioeconómica extrema (coeficientes de Gini mayores de .60).

A pesar de las características específicas de estos regímenes de bienestar, Rodríguez Enriquez (2012) comenta que la autora identifica dos regularidades: por un lado, todos los regímenes son informales y, por otro, el trabajo femenino no remunerado es fundamental para la producción y el acceso al bienestar.

Esta tipología desarrollada por Martínez Franzoni nos invita a pensar las características de la producción de bienestar en Argentina. El modelo de familia basado en el hombre proveedor atraviesa todos los regímenes de bienestar. Históricamente, tal como dijimos más arriba, en Argentina el conjunto de los derechos sociales nace como un derivado de los derechos laborales. Los principales seguros sociales cubrían a los trabajadores (los cuales eran principalmente hombres), lo que produjo una diferenciación de hecho y de derecho para con las mujeres.

De esta manera, en nuestro país el acceso al bienestar está mediado por la posición de las personas en el mercado laboral y su nivel de ingresos, enmarcado en una concepción individual del sujeto que aporta a su bienestar. En este sentido, la desmercantilización del bienestar se dirige selectivamente a los sectores de menores ingresos, mientras que en el resto de la población es mínima. Si a esto le sumamos la importancia fundamental que tiene el trabajo femenino no remunerado, podemos decir que Argentina, según la tipología de Martínez Franzoni, es un régimen de bienestar liberal de proveedor único.

Si la ciudadanía se refiere a la participación individual y colectiva en las instituciones creadas por las sociedades capitalistas, apoyándose en el binomio inseparable de (in)dependencia económica e (in)dependencia de cuidado, entonces las mujeres han tenido históricamente un acceso diferencial al ejercicio de la ciudadanía. La división sexual del trabajo entre hombres proveedores y mujeres cuidadoras, esposas y madres, combinada con la falta de incentivos de la mujer para ingresar al mercado de trabajo, convirtió a la mujer en una ciudadana de segunda. El cuidado, al quedar recluido a la esfera de lo privado, se volvió invisible. Quedó detrás del telón, como algo pre-ciudadano (Martínez Franzoni 2005).

\section{El cuidado entra en crisis}

Con el telón de fondo de las transformaciones del capitalismo global en las sociedades de bienestar, se asiste a una reconfiguración de la provisión de los cuidados que varias autoras denominan "crisis de cuidados". Las tensiones que aparecen se derivan tanto del nuevo papel de las mujeres en las sociedades de finales del siglo XX como en los 
cambios en los contenidos, protagonistas y circunstancias en las que el trabajo de cuidado se da.

Una de las transformaciones más notables de los últimos cincuenta años en América Latina es la creciente participación de las mujeres en el mercado laboral. Durante la década pasada y las anteriores, la tasa de entrada de las mujeres a la fuerza laboral creció aceleradamente (0,9 puntos porcentuales por año). En 2002 la tasa de participación laboral femenina alcanzó el 62\%, llegando al 65\% en el año 2012 (Gasparini y Marchionni 2015).

En paralelo a esta irrupción de la mujer en el mercado laboral, la carga de trabajo no remunerado no disminuyó. De acuerdo a un informe realizado en el 2013 por el Equipo Latinoamericano de Justicia y Género (ELA), con base en la Encuesta de Hogares Urbanos (EAUH) del INDEC, las mujeres argentinas dedican casi el doble de horas que los hombres al trabajo doméstico. Esto se acentúa en los sectores de menores ingresos, ya que estas mujeres son las que más tiempo destinan al trabajo no remunerado y enfrentan las mayores demandas de cuidado, al mismo tiempo que acceden a débiles oportunidades de trabajo remunerado. ${ }^{6}$ De esta manera, las mujeres acceden al ámbito público sin menguar su protagonismo como cuidadoras en el ámbito doméstico. Así, la crisis de cuidado se convierte en un concepto estratégico, que logra iluminar no sólo los cambios demográficos y sociales, sino también económicos y políticos, característicos de la sociedad actual, y la manera en la que impactan en las necesidades y en la provisión de cuidados (Carrasquer Oto 2013).

\section{De la "presencia/ausencia" a la "doble presencia/ausencia"}

En el modelo previo de reparto de los cuidados, las mujeres tenían una "presencia- ausencia": aseguraban la realización del trabajo de cuidado necesario para la gestión cotidiana de la vida que, siguiendo a Pérez Orozco, era la base invisibilizada del iceberg que sustentaba el conjunto de la estructura. Para no caer en una cómoda lectura con sesgo clasista, es necesario pensar al sujeto femenino del trabajo doméstico como un sujeto colectivo: cuando las mujeres debían salir al mercado laboral, otras redes de mujeres las reemplazaban en la casa. De esta manera, la presencia/ausencia femenina era una experiencia social.

En este nuevo escenario, este modelo se resquebraja y cruje por todos lados. La presencia cada vez más masiva de las mujeres en el mercado laboral transforman la experiencia de la presencia/ ausencia en una doble presencia/ausencia. Este concepto pretende captar "ese estar y no estar en ninguno de los dos lugares [el mercado y el trabajo de cuidados no remunerados], y el sufrimiento y limitaciones que tal situación comporta" (Izquierdo 1998 apud Pérez Orozco).

De esta manera, la otra cara de la doble presencia femenina es la triple ausencia de los Estados, los mercados y los hombres. En realidad el mercado (las empresas privadas), está ganando terreno en el ámbito de los cuidados, que se convierte en un nuevo espacio de donde obtener rédito económico. Las mujeres conquistamos e intervenimos activamente 
en nuevos espacios, pero, en regímenes de bienestar como los latinoamericanos, sólo el mercado está dispuesto a reemplazar los trabajos de cuidado que garantizábamos.

Volvamos al caso de Laura que ilustró el inicio de este trabajo. A pesar que ella es una profesional inserta en el mercado laboral formal, no tiene acceso a un cuidado infantil provisto por el Estado, debido a la escasez de guarderías públicas. Esta baja cobertura de salas maternales, guarderías y jardines de infantes se fundamenta en el supuesto de que el cuidado de niñas y niños debe ser provisto por las familias cuando aún no están en edad de ingresar a la educación formal (Esquivel 2012). De esta manera, para desfamiliarizar ese cuidado debe acudir al mercado o, de lo contrario, y tal como lo resolvió en este momento, gestionarlo de manera intrafamiliar pidiéndole ayuda a las abuelas, que también son mujeres. Entonces, una vez más, no sólo se resuelve dentro del seno familiar, sino que todo queda entre chicas.

Los ámbitos en los cuales se gestiona la vida siguen siendo invisibles, y se superponen dos lógicas que no encuentran como conciliarse. La vida cotidiana se convierte así en un espacio de tensiones, angustias, negociaciones y sororidades que intentan resolver los conflictos que emergen al no modificarse la distribución social del cuidado. Estos conflictos permanecen encerrados en el ámbito de lo privado y no se plantean como una demanda de ciudadanía de género que vuelve necesario y urgente el "descongelamiento" de la rígida división por géneros del trabajo y de las responsabilidades.

Estas estrategias, que son privadas pero políticas, terminan resolviéndose en la línea femenina hijas-madres-abuelas. Laura tuvo que recurrir a las abuelas de su hija para que la cuiden mientras ella trabaja. Bajo una fachada rosada de amor y altruismo, estas estrategias también esconden relaciones de poder, a veces de maltrato y de malestar, de interdependencia obligatoria.

En este escenario, hablar sobre el cuidado es estratégico. Nos permite dar cuenta por un lado de la experiencia de vida de las mujeres y, por otro, es una categoría de análisis muy potente para el estudio de las políticas sociales. Esto implica pensar al cuidado como parte de una organización social, es decir que, una vez más, los límites entre lo privado y lo público se vuelven difusos, se expanden, se convierten en territorios de disputa, y nos obliga a mirar de qué manera se produce y se organiza el cuidado en una sociedad determinada y qué efectos tiene en la configuración de las relaciones sociales y de género (Faur 2014).

Los Estados, mercados y familias intervienen en la provisión de bienestar, pero no hay una única manera de organizar el trabajo de cuidados en esta tríada. Los análisis en torno a la organización social del cuidado permiten conocer mejor cómo se distribuye el trabajo de cuidados en ese triángulo socioinstitucional, así como las normas y valores que pautan y reproducen un desigual reparto de tareas y responsabilidades de cuidados. 
La discusión sobre el cuidado como parte de una organización social, como algo que no le pertenece sólo a las familias, forma parte de la invitación a pensar la necesidad de una "nueva arquitectura" del bienestar. Cuando Hannah Arendt (1993) retoma la frase de los Evangelios "Nos ha nacido un niño" ("A child has born into us”), lo que nos está diciendo es que un nacimiento no es sólo un engendrar, el multiplicarse de la especie humana, sino que es un nacimiento que se da en una comunidad pre-existente (el $u s)$.Y esa comunidad, que se revela en el acto mismo del nacimiento, debe hacerle espacio al recién llegado/a.

En este sentido, considerar los cuidados como derecho de ciudadanía apela a la idea de que la responsabilidad social de los cuidados es colectiva y no individual. Implica correr el telón pre-ciudadano y sacarlos a escena. El desafio, entonces, es lograr que esta responsabilidad se distribuya de manera equitativa y que el peso de los cuidados no recaiga sólo sobre los colectivos menos favorecidos (Carrasquer Oto 2013).

En esta disputa, es conveniente explorar la propuesta de Pérez Orozco (2006) de reconocer la interdependencia social. Para la autora, este reconocimiento es una apuesta política. Ya se ha demostrado que la distinción entre autonomía/ dependencia se erigió tanto sobre la negación de los trabajos no remunerados como la asimetría de las relaciones de género, que construyeron una subjetividad femenina dispuesta para el cuidado y una subjetividad masculina que espera ser cuidada. Si sólo ampliamos la definición de autonomía de modo que incluya el trabajo de cuidados no remunerados, lo único que estaríamos haciendo es desplazar el eje que divide a la sociedad entre personas autónomas y dependientes.

De esta manera, una vez más, la dependencia se convierte en una situación estática e individualizada y se esconden las propias necesidades y dependencias de quienes cuidan. Se actualiza una relación asimétrica entre quienes necesitan cuidados y quienes otorgan cuidados. En este desplazamiento, dice Pérez Orozco, se pierde la oportunidad de cuestionar al individuo autónomo, sujeto fetiche del liberalismo, y de reclamar la interdependencia social que, en definitiva, es lo que caracteriza nuestra condición humana.

En palabras de la autora, la apuesta, entonces, "se situaría en la reclamación de la interdependencia, rebelándonos contra la asunción de un modelo de autosuficiencia que resulta frustrante (...), que establece relaciones de asimetría (...), y oculta las voces y las contribuciones de quienes son etiquetados/as como dependientes" (Pérez Orozco 2006:13). Esta vuelta de tuerca permite entender que tanto las necesidades de cuidado como las responsabilidades de brindar cuidados están ideológica y socialmente construidas.

Esto implicaría reclamar una "lógica ecológica del cuidado", o una "ética del cuerpo social" que nos permita romper con el 'círculo vicioso' de los cuidados, dejando de lado las mistificaciones que lo relacionan con el altruismo, la familia y el amor. Nos convierte en sujetos activos que reclaman el derecho a unos servicios de cuidados accesibles y 
profesionalizados al mismo tiempo que reconocen material y simbólicamente el valor de los cuidados como pieza clave para el bienestar y la reproducción social.

\section{De la ciudadanía a la cuidadanía}

Hace poco Laura tuvo una arritmia nerviosa. Cuenta que sentía como que "un elefante le pisaba el pecho y no la dejaba respirar". Le dijeron que fue por stress. Ella se lo atribuye al trabajo, porque trabaja en el área de Recursos Humanos de una empresa y muchas personas le "cuentan sus problemas". Cuidar también es escuchar los problemas de los otros.

Laura es joven y sana, por lo que este episodio no debería volver a repetirse y sólo fue un susto. Pero también podemos preguntarnos si la doble presencia/ ausencia, que modela una wonderwoman que puede con el trabajo tanto dentro como fuera de la casa, característico de la mujer en la actualidad, le genera esas reacciones.

¿Cuál es el impacto en la salud y el bienestar que los cuidados tienen en las mujeres? ¿Alguien cuida a las cuidadoras?

En el prefacio de Las palabras y las cosas (2014), Foucault citaba un texto de Borges que nos muestra, de golpe, el límite de nuestro propio pensamiento. Hay cosas que son imposibles de ser pensadas porque no tenemos palabras para nombrarlas. Sabiendo esto, Pérez Orozco, ante el agotamiento de la idea de ciudadanía propia del Estado de Bienestar liberal-democrático, se hace eco de la propuesta de la cuidadanía, que se enmarca en la revisión de la ciudadanía desde la óptica de los cuidados.

Este concepto no es un simple juego de palabras ni es la versión feminista de la ciudadanía. La cuidadanía rompe con la noción de ciudadanía porque pone en el centro el cuidado de la vida y reemplaza el ideal de autonomía por un reconocimiento de la interdependencia social. Los problemas y el dolor del otro también nos afectan por ser parte de una comunidad. La cuidadanía, entonces, es una invitación a pensar otras maneras de relacionarnos, a imaginar nuevas formas de estar en el mundo.

\section{El cuidado como herramienta política}

Teniendo en cuenta este recorrido, podríamos afirmar que el cuidado es un concepto potente y estratégico. El cuidado es trabajo y es relación interpersonal, pero también es una responsabilidad socialmente construida que se inscribe en contextos sociales y económicos particulares. Este enfoque nos permite enlazar la mirada más microsocial (los roles de género que hacen recaer el cuidado en las mujeres) con los modos particulares en los que el estado regula y moldea la prestación de estos cuidados (Esquivel 2012). De esta manera, distintos regímenes de bienestar se asocian a distintos regímenes de cuidado. 
Según Esquivel, para caracterizar un régimen de cuidado interesa saber dónde se cuida, quién cuida y quién paga los costos de ese cuidado.

Este enfoque innovador nos permite realizar un "corte transversal" sobre los distintos pilares del bienestar (familias, estado, mercado y comunidad) para poder entender su participación relativa en la provisión de cuidado. Al mismo tiempo, el análisis feminista de las políticas públicas visibiliza los supuestos que subyacen a su diseño y aplicación respecto de los roles de las mujeres y la familias en la provisión de cuidados.

Es importante remarcar que debido al acceso diferencial al bienestar característico de las sociedades latinoamericanas, de acuerdo a la participación en el mercado laboral y el nivel de ingresos, no es posible hablar de un único régimen de cuidado. De todas maneras, a pesar de esta heterogeneidad, la autora identifica algunos rasgos comunes que caracterizan a la organización social del cuidado en América Latina:

1) El cuidado sigue estando en manos de las familias y, más específicamente, en las mujeres.

2) Se verifica un acceso diferencial al cuidado que refleja las desigualdades de ingresos.

3) Los intentos por conciliar a la familia y al trabajo son muy débiles y sólo impactan en el sector formal del mercado laboral.

Para la autora, que el cuidado pase de ser un concepto con potencialidad analítica a una herramienta política depende de clarificar las agendas del cuidado que están vigentes actualmente en América Latina. Una de las propuestas, debatidas en países tales como Ecuador, Venezuela y Paraguay, es la remuneración al cuidado. De esta manera, se equipara el trabajo doméstico y de cuidados de las amas de casa al trabajo doméstico remunerado, otorgándoles el derecho a la seguridad social. De acuerdo a diferentes autores (Dobrée, González, Soto 2011; Pautassi, Arcidiácono y Straschnoy 2014), estas propuestas tienden a subrayar estereotipos de género, impactando en el retiro del mercado de trabajo por parte de las mujeres más pobres. Por otro lado, las amas de casa sin participación en el mercado de trabajo son un 'actor político' que va disminuyendo su proporción entre las mujeres y familias jóvenes.

Otra de las propuestas es la redistribución del cuidado. Este planteo se enmarca en la línea que trabajamos más arriba, que considera al cuidado como responsabilidad compartida no sólo entre varones y mujeres, sino entre las familias y la esfera pública. La redistribución del cuidado invita a deconstruir la norma del "trabajador ideal" pensado como un "hongo de Hobbes", que brota libre de cargas o necesidades de cuidado, plenamente disponibles para el empleo (Pérez Orozco 2006). Así, el cuidado puede empezar a dejar de ser una "cosa de mujeres", inherente a la subjetividad femenina, y comenzar a ser una actividad en la que participen todos los sectores encargados de la producción del bienestar. 


\section{¿Cómo hacemos para cuidar a este bebé?}

Hasta ahora, la historia de Laura nos ayudó a mirar de qué manera se distribuye el cuidado y la producción de bienestar en Argentina. Más específicamente, vamos haciendo tímidos intentos de pensar el cuidado infantil en nuestro país. Recapitulando, de acuerdo a la tipología elaborada por Martínez Franzoni (2005), Argentina presenta un régimen liberal de proveedor único, en el cual el acceso al bienestar está mediado por la posición de las personas en el mercado laboral y su nivel de ingresos.

En este sentido, si observamos la combinación específica de la tríada de bienestar se verifican distintos niveles de desmercantilización y desfamiliarización, de acuerdo al estrato social. Las políticas sociales de los últimos años apuntaron selectivamente a desmercantilizar el acceso al bienestar de los sectores de menores ingresos pero, a medida que se asciende en la pirámide socioeconómica, la desmercantilización disminuye. El grado de desfamiliarización es mucho más bajo todavía. En relación con el cuidado infantil, la noción de desfamiliarización permite observar el grado en que las políticas públicas facilitan la provisión y el acceso a servicios de cuidado, redistribuyen la función social del cuidado entre distintas instituciones públicas y privadas y superan - o no - la visión según la cual las familias (y dentro de estas, las madres) serían las responsables exclusivas de proveer cuidado.

En Argentina la oferta pública es muy reducida. Por ejemplo, existen guarderías gratuitas para niños y niñas menores de dos años, pero las vacantes no cubren la totalidad de la población. Algo similar ocurre con la sala de tres años, que actualmente es obligatoria, pero aún no se construyeron suficientes jardines que garanticen el acceso de todos los niños y niñas a la educación formal. ${ }^{7}$ De esta manera, las familias más pobres deben hacerse cargo de los más pequeños y las familias con ingresos más altos acceden a la desfamiliarización a costa de un incremento de su mercantilización.

También vimos cómo los supuestos que subyacen a la idea de proveedor único y al familismo implícito configuran las relaciones de cuidado dentro de la familia, recayendo este trabajo en las mujeres de diferentes generaciones. Aún en los sectores de ingresos medios, el alto costo de la desfamiliarización vuelve necesario diseñar estrategias de cuidado que incluyan a otros miembros de la familia, generalmente mujeres.

Hasta acá, el caso de Laura nos permitió dar un pantallazo de cómo se configura la vida de una mujer de clase media, profesional y madre primeriza, a partir de la distribución y asignación de responsabilidades de cuidado. Pero en Argentina Laura pertenece a una minoría de mujeres afortunadas. Así que debemos ampliar la mirada y analizar cómo hacen otras mujeres para gestionar el cuidado de los niños y niñas. 


\section{Enfocando la mirada: la Asignación Universal por $\mathrm{Hijo/a}$}

En el contexto latinoamericano de un giro hacia una "nueva izquierda", que transformó el rol del Estado apostando a una nueva etapa posneoliberal, se afirmaron derechos, valores, la esfera pública y la ciudadanía (Sader 2008). Desde 2003, la puesta en marcha de un nuevo modo de desarrollo en Argentina estuvo signada por elevadas tasas de crecimiento, mejoras en la dinámica del mercado de trabajo y por la configuración de un nuevo paradigma del sistema de seguridad social más equitativo, aunque sin resolver por completo la situación de segmentos poblacionales en situación de vulnerabilidad.

Estos procesos de transformaciones se dieron en un marco en el cual la orientación general de los programas sociales comenzó a acotar su accionar al campo de la educación y la salud y a intervenir en tres poblaciones específicas (niños, adolescentes y mujeres embarazadas). De acuerdo a Shahra Razavi (2007), las políticas posneoliberales promueven principalmente las capacidades de la infancia, entendidas como una inversión en "capital humano". Este enfoque de inversión social que promueve un bienestar activo, es propuesto por países europeos y la OCDE, y se contrapone con el enfoque de bienestar pasivo de la posguerra, que es visto en términos negativos por haberse orientado a promover el consumo y es acusado de fomentar la "dependencia".

En el 2009 se creó la Asignación Universal por Hijo para Protección Social (AUH), y dos años más tarde la Asignación Universal por Embarazo (AUE), lo cual abrió una nueva etapa para las políticas sociales argentinas. La "arquitectura" del bienestar se modificaba y el régimen de asignaciones familiares pasó a incluir a sectores de trabajadores informales y desempleados que históricamente habían sido excluidos de esta prestación. Este hecho, según Faur, "marcó un nuevo territorio simbólico y material en las políticas sociales", ya que resignificó la noción de universalidad en las políticas sociales.

Es por esto que existen discusiones sobre qué es la AUH, ya que está situada en un espacio intermedio entre una asignación familiar tradicional de los sectores formales y un programa de transferencias condicionadas. ${ }^{8}$ No puede ser considerada una transferencia condicionada en el sentido estricto debido a su inserción en el sistema institucional (implicó una reforma del sistema de seguridad social), su apertura permanente y la forma en que es oficialmente presentada (Pautassi, Arcidiácono y Straschnoy 2014).

La AUH establece normativamente que el ingreso lo recibe uno solo de los progenitores. En los considerandos del decreto 614/13 del Poder Ejecutivo Nacional, cuyo artículo 7 establece que son las madres quienes tienen prioridad para cobrar la AUH, se señala:

Que la mujer es uno de los pilares fundamentales en el que se apoya la familia y la sociedad, teniendo un rol 
fundamental en el cuidado de los hijos.

Que dicha condición la hace esencial al momento de ser la receptora de los recursos otorgados por la Seguridad Social para dar cobertura a los niños, adolescentes y personas con discapacidad.

Los varones solo están contemplados en ocasión de que tengan hijos bajo su cuidado por guarda, tenencia individual o fallecimiento de la madre. De este modo, desde el Estado se refuerza la idea de que las mujeres son, en tanto madres, las principales responsables de las tareas familiares de cuidado. Así, por medio de regulaciones y políticas se fortalece la división sexual del trabajo que impera en las sociedades latinoamericanas.

Esta decisión también se enmarca en numerosos estudios que, desde la década de 1990, demostraron que las mujeres son "mejores administradoras" de los recursos que los hombres al asignarlos al bienestar de sus hijos. De esta manera, y siguiendo a Faur (2014) y Molyneux (2001), las madres son las intermediarias elegidas por los Estados para garantizar el bienestar de la población infantil. En este movimiento, vuelve a surgir la pregunta por la ciudadanía de las mujeres y por la democratización de las relaciones de género. Sin embargo no debemos invisibilizar en este análisis las voces de las propias mujeres receptoras. La investigación realizada por Pautassi, Arcidiácono y Straschnoy (2014) recoge en sus entrevistas una valoración positiva del impacto que tiene en la autonomía de estas mujeres percibir un ingreso propio.

Al mismo tiempo, al no vincularse con otros programas que faciliten el ejercicio de cuidado que se les impone a las madres (con excepción del plan NACER-SUMAR que brinda atención, entre otros, a embarazadas, niños, niñas y adolescentes), las condicionalidades de la AUH son, muchas veces y especialmente para la población de menores ingresos, difíciles de cumplir. Pautassi, Arcidiácono y Straschnoy (2014) recogen las quejas de las madres fundamentalmente sobre los diversos tipos de obstáculos en relación con la obtención de turnos médicos y/o vacantes escolares. Esto se enmarca en una problemática más amplia: el desigual acceso a la seguridad social y a los servicios sociales, lo que produce impactos diferenciados según la pertenencia socioeconómica de las familias.

\section{Palabras finales}

En este trabajo hemos esbozado algunas líneas acerca de la organización del cuidado infantil en la Argentina. Para eso, fue necesario revisar cómo se produce y distribuye el bienestar en nuestro país. También, problematizamos y conceptualizamos el tema del cuidado, el cual se vuelve un concepto fundamental para el análisis de las políticas sociales. Luego, retomamos varias investigaciones para observar la Asignación Universal por Hijo (AUH) en su complejidad, preguntándonos por los supuestos relacionados con el cuidado y el rol de las mujeres que subyacen a esta política pública. 
Para finalizar, nos interesa preguntarnos sobre cuál es el efecto de este encasillamiento de las mujeres en el espacio de lo doméstico en relación a su participación en el mercado laboral. En estas páginas mencionamos el crecimiento en la participación laboral femenina. Pero desde principios del 2000 este crecimiento se ha desacelerado: el aumento fue de apenas 0,3 puntos porcentuales. Según una investigación realizada por el Centro de Estudios Distributivos, Laborales y Sociales (CEDLAS) de la Universidad Nacional de La Plata (2015), este patrón está presente en todos los grupos de mujeres pero sobre todo en las mujeres casadas o de contextos socioeconómicos más vulnerables. Según los autores, emerge un escenario dual en el que la participación laboral de las mujeres urbanas con alta educación es más alta y crece, mientras que la participación de mujeres con niveles de educación más bajos se estanca.

La AUH no es una política de conciliación de familia y trabajo, pero tiene efectos específicos en las vidas de las mujeres y en el modelado de las relaciones de género. Cuando la legislación no acompaña o legitima la posibilidad de transformar las jerarquías de género, estos cambios no se institucionalizan y termina persistiendo un sistema de género extemporáneo.

De todas maneras, las grietas en las estructuras son cada vez más visibles. El 14 de junio de 2018, cuando el proyecto de Ley de Interrupción Voluntaria del Embarazo obtuvo media sanción en la Cámara de Diputados, las diputadas Silvia Lospennato, Victoria Donda y Carla Carrizo participaron del programa de radio de María O`Donnell (2018). Cuando la conductora les preguntó qué otros puntos figuraban en la agenda, las diputadas coincidieron en que es necesaria una "Ley Nacional de Cuidado". Esto permitiría "devolverle tiempo" a las mujeres para que lo utilicen en el desarrollo de sus carreras y sus profesiones.

Acompañando el planteo de Rodríguez Enríquez (2012), creemos que urge la necesidad de permitirnos imaginar un sistema de protección social integral, en el marco del cual las estructuras que perpetúan las desigualdades de clase y de género sean desafiadas y erosionadas. De esta manera, los roles de la tríada del bienestar necesitan ser discutidos para avanzar hacia sistemas de protección social con coberturas universales y homogéneas: un estado cuidadoso del bienestar de todos y todas. 
${ }^{1}$ El caso de Laura forma parte de una investigación en curso que sigue las trayectorias de mujeres y sus estrategias con respecto al cuidado de los/as niños/as. En este caso, Laura es una mujer de 33 años que vive en la Ciudad Autónoma de Buenos Aires. Tiene una licenciatura en Recursos Humanos y trabaja en el área de Administración de Personal de una PyME, en relación de dependencia. Está en pareja y convive con el padre de su primera hija, quien también trabaja en relación de dependencia y es propietario de dos taxis. El nombre de Laura es ficcional.

${ }^{2}$ Esta prestación cubre a 3.956.042 niños, niñas y adolescentes menores de 18 años que viven en hogares cuyos adultos responsables son desocupados, trabajadores informales con un salario inferior al mínimo vital y móvil, trabajadores del servicio doméstico y monotributistas sociales (ANSES 2017). Las receptoras de la Asignación Universal son mayoritariamente mujeres (97, 96\%).

${ }^{3}$ En sus discursos de campaña electoral de 1983, Raúl Alfonsín, el primer presidente de la transición en Argentina, sintetizaba esta ilusión: "con democracia no sólo se vive, con democracia se educa, con democracia se come, con democracia se sana". ${ }^{4}$ Entre 1990 y 2000 la brecha de género en la tasa de actividad pasa del 49,32\% al 39,56\%, en contraposición a la brecha en la tasa de desempleo que pasó de 5,81\% al 16,50\%. Luego de la profunda crisis política, económica e institucional que tuvo el país en 2001/2, los hombres presentaron mayores niveles de desocupación que las mujeres (DINREP 2015).

${ }^{5}$ Desde hace algunos años en América Latina y el Caribe se realizan Encuestas de Uso de Tiempo (EUT), con el objetivo de reconocer las situaciones de desigualdad en la composición del trabajo doméstico y entregar una visión más real de la organización social. De esta manera, las EUT proporcionan datos útiles para elaborar y apoyar la implementación de políticas públicas más eficaces para la reducción de las desigualdades. Sin embargo, explica Marco Navarro (2012), la integración de sus resultados en las políticas públicas es incipiente o no se ha concretado. ${ }^{6}$ Mientras que las mujeres del quinto quintil de ingresos le dedican 3 horas diarias al TNR, las mujeres del primer quintil le dedican casi el triple: 8,1 (Rodríguez Enríquez 2012).

${ }^{7}$ Según un informe del Observatorio Argentino por la Educación, basado en datos oficiales, uno de cada tres chicos que asiste al jardín hoy en el país (el 32,6\%) lo hace en uno privado. A medida que disminuye la edad de los/as niños/as, este porcentaje aumenta: en sala de 2, por ejemplo, 6 de cada 10 chicos van a jardín privado. Y entre los de 45 días a 2 años, más de la mitad (Braginski 2018).

${ }^{8}$ Los programas de transferencias condicionadas son implementados por la gran mayoría de los países latinoamericanos como la principal forma de política social para atender el problema de la pobreza estructural y asegurar un nivel básico de ingresos monetarios. Dichos programas han tenido entre sus principales objetivos el apoyo a las familias para mejorar sus condiciones de vida y la inversión en "capital humano" a través de responsabilidades asumidas por sus beneficiarios en la asistencia en salud y educación. Concretamente, estas transferencias condicionales otorgan un beneficio económico en forma de efectivo a los beneficiarios, y éstos, como contraparte, satisfacen una serie de condiciones que tienen como objetivo, en el corto plazo, reducir la pobreza, y en el largo plazo, eliminar la pobreza inter-generacional (Cacace y Albornoz 2012). 


\section{Referencias bibliográficas}

Arendt, Hannah (1993) La condición humana. Barcelona / Buenos Aires: Paidós.

Cacace, Alejandro y Pablo Albornoz (2012) Fiscalización de los planes sociales en Argentina. Buenos Aires: Auditoría General de la Nación. http://www.olacefs.com/ wp-content/uploads/2014/06/DOC_10_10_2012_ContribucionesTemaTecnicoIII_ Reduccion_P.pdf accesado el 1 de febrero 2018.

Carrasquer Oto, Pilar (2013) "El redescubrimiento del trabajo de cuidados: algunas reflexiones desde la sociología”. Cuaderno de Relaciones Laborales 31(1): 91-113. http:// dx.doi.org/10.5209/rev_CRLA.2013.v31.n1.41633 accesado el 22 de diciembre 2017.

Cavarozzi, Marcelo (2017) La soledad de la democracia en la América Latina contemporánea. Conferencia Magistral, Universidad Nacional de San Martín, septiembre.

Dirección de Relaciones Económicas con las Provincias (2015) Características de la fuerza de trabajo femenina. Las diferencias respecto del hombre. Ministerio de Economía y Finanzas Públicas de la Nación Argentina, Febrero. http://www2.mecon.gov.ar/hacienda/dinrep/Informes/archivos/Caracteristicasdelafuerzadetrabajofemenina.pdf accesado el 22 de diciembre 2017.

Draibe, Sonia y Riesco, Manuel (2006) Estado de bienestar, desarrollo económico y ciudadanía: algunas lecciones de la literatura contemporánea. México: CEPAL, Serie Estudios y Perspectivas 55. http://dds.cepal.org/eventos/presentaciones/2006/0907/Sonia_ Draibe_EstudiosCEPALMexico.pdf accesado el 21 de diciembre 2017.

Dobrée, Patricio, Myrian González y Clyde Soto (2011) La migración femenina paraguaya en las cadenas globales de cuidados en Argentina: transferencia de cuidados y desigualdades de género. Paraguay: ONU Mujeres.

Esping-Andersen, Gøsta (1993) Los tres mundos del Estado de Bienestar.Valencia: ediciones Alfons el Magnanim-Generalitat Valenciana.

Esquivel, Valeria (2012) "Cuidado, economía y agendas políticas: una mirada conceptual sobre la organización social del cuidado en América Latina”, en V. Esquivel (ed.) La economía feminista desde América Latina: Una hoja de ruta sobre los debates actuales en la región. Santo Domingo: ONU - Mujeres, 2012: 141-182. http://www.redetis.iipe.unesco. org/wp-content/uploads/2013/07/Economia-feminista-desde-america-latina-1.pdf accesado el 21 de diciembre 2017. 
Faur, Eleonora (2014). El cuidado infantil en el Siglo XXI. Buenos Aires: Siglo Veintiuno.

Foucault, Michel (2014) Las palabras y las cosas. Una arqueología de las ciencias humanas. Buenos Aires: Siglo XXI editores.

Gasparini, Leonardo y Marchionni, Mariana (eds.) (2015) Bridging gender gaps? The rise and deceleration of female labor force participation in Latin America, La Plata: Universidad Nacional de La Plata.

Marco Navarro, Flavia (2012) La utilización de las encuestas de uso del tiempo en las políticas públicas. Santiago: CEPAL, Serie Mujer y desarrollo 119. https://repositorio.cepal.org/ bitstream/handle/11362/5849/1/S1200884_es.pdf accesado el 24 de junio de 2018.

Martínez Franzoni, Juliana (2005) "Regímenes de bienestar en América Latina: consideraciones generales e itinerarios regionales". Revista Centroamericana de Ciencias Sociales, 4 (2): 1-32. http://www.derechoshumanos.unlp.edu.ar/assets/files/documentos/regimenes-de-bienestar-en-america-latina-consideraciones-generales-e-itinerarios-regionales.pdf accesado el 20 de diciembre 2017.

Millet, Kate (1995) Política sexual. Madrid: Cátedra.

Molyneux, Maxine (2001) “Género y Ciudadanía en América Latina: cuestiones históricas y contemporáneas”. Debate Feminista 12 (23):3-66. http://www.debatefeminista.pueg.unam. mx/wp-content/uploads/2016/03/articulos/023_01.pdf accesado el 22 de diciembre 2017.

Pautassi, Laura, Pilar Arcidiácono, y Mora Straschnoy (2014) "Condicionando el cuidado. La Asignación Universal por Hijo para la Protección Social en Argentina”. Íconos. Revista de Ciencias Sociales 50: 61-75. http://revistas.flacsoandes.edu.ec/iconos/ article/view/1429

Pérez Orozco, Amaia (2006). “Amenaza Tormenta: la crisis de los cuidados y la reorganización del sistema económico”. Revista de Economía Crítica 5: 7-37.

Rodríguez Enríquez, Corina (2012) "Políticas de atención a la pobreza y las desigualdades en América Latina: una revisión crítica desde la economía feminista”, en Esquivel, Valeria (ed.) La economía feminista desde América Latina: Una hoja de ruta sobre los debates actuales en la región. Santo Domingo, ONU - Mujeres, 2012:141-182. http:// www.redetis.iipe.unesco.org/wp-content/uploads/2013/07/Economia-feministadesde-america-latina-1.pdf accesado el 5 de febrero 2018. 
Rodríguez Enríquez, Corina (2013) "El trabajo de cuidado no remunerado en Argentina: un análisis desde la evidencia del Módulo de Trabajo no Remunerado", Documentos de Trabajo "Políticas públicas y derecho al cuidado". Buenos Aires: Equipo Latinoamericano de Justicia y Género. Disponible: http:// elcuidadoenagenda.org.ar accesado el 20 de diciembre 2017.

Sader, Emir (2008). "Refundar el Estado”. Posneoliberalismo en América Latina. Buenos Aires: CTA- CLACSO.

Razavi, Shahra (2007) “The political and social economy of care in a development context. Conceptual Issues, Research questions and policy options”. Gender and development 3: 1-39. http://www.unrisd.org/80256B3C005BCCF9/(httpAuxPages)/2DBE6A93350A 7783C12573240036D5A0/\$file/Razavi-paper.pdf accesado el 5 de febrero 2018.

Sunkel, Guillermo (2007) "Regímenes de bienestar y políticas de familia en América Latina”, en Arriagada, Irma (ed.) Gestión y financiamiento de políticas que afectan a las familias. Santiago de Chile: CEPAL Serie Seminarios y Conferencias 49:67-76.

\section{Fuentes periodísticas}

Braginski, Ricardo (12 de junio de 2018) "Uno de cada 3 chicos que van al jardín de infantes lo hace en uno privado". Clarín. Recuperado de https://www.clarin.com/ sociedad/chicos-van-jardin-infantes-hace-privado_0_SJu5NyAeX.html

O`Donnell, María (conductora) (14 de junio de 2018) Tarde para nada. Buenos Aires: Radio con vos.

\section{Como citar este artículo:}

Cánovas Herrera, Gisela (2018) “Las mujeres y los regímenes de bienestar. Una mirada feminista para el debate de la organización social del cuidado en Argentina”. Revista Perspectivas de Políticas Públicas vol. 8 No15:67-87 